\title{
KÖNNTE EINE FRAU KATHOLISCHER PRIESTER WERDEN?
}

In den letzten Jahren läuft eine lebhafte Diskussion betreffs der Möglichkeit einer Zulassung von Frauen zum Priesteramt in der katholischen Kirche. Im Zusammenhang mit der Anvertrauung der Priesterfunktionen an Frauen in einigen abgetrennten Kirchen, nehmen an dieser Diskussion nicht nur Theologen, sondern auch Frauen teil ${ }^{1}$. Das Problem der Anteilnahme von Frauen am sakramentalen Priestertum der katholischen Kirche betrachtet J. Galot als Ausschnitt eines weiteren Problems, nämlich der Stellung der Frau innerhalb der Kirche. Als Konsequenz der Anerkennung der Gleichheit von Frauen und Männer im staatlichen und sozialen Leben wurde zum ersten Mal das Problem dieser Gleichheit in allen kirchlichen Funktionen und insbesondere im sakramentalen Priesteramt aufgestellt ${ }^{2}$.

Es wird die Diskussion unter Theologen und Nichttheologen geführt, es werden Argumente pro und contra erwogen, unterdessen aber wird vom Magisterium Ecclesiae die traditionelle Lehre laut Kan. 1023 CJC aufrecht erhalten, dass nur ein gewachsener, getaufter Mann, mit entsprechender Intention, ein Subjekt der Priesterweihe bilden kann. Dieser Standpunkt der Kirche wurde schon im Jahre 1970 bestätigt, als die Kongregation für die Glaubenslehre ein Dekret erliess, wonach im Einklang mit dem Traditionellen Grundsatz der Kirche der Dienst am Altar Frauen nicht gestattet ist ${ }^{3}$. Manche Theologen sind gegenwärtig der Meinung, dass trotz eines derartigen Standpunktes der Kirche die Frage einer Zulassung von Frauen zur Priesterweihe definitiv noch nicht erledigt und die Diskusion nicht beendet ist, da der Theologe nach der Ursache und Begründung dieser kirchlichen Praxis sucht. Deshalb erheben sich Stimmen des Zweifels an der Richtigkeit der steifen Haltung des Magisterium Ecclesiae und es wird nach Argumenten gesucht, die eine Möglichkeit der Zulas-

${ }^{1} \mathrm{Vgl}$. Les femmes d'aujourd'hui et l'Eglise, „Le Supplément” 127:1978. Die von Grauen inspirierte Bewegung „Vie Nouvelle" richtet sich gegen angeblichen Antifeminismus der Kirche und gegen das Dekret der Glaubenskongregation. Siehe hiezu: M. B o u d o n, Hommes et femmes vers une „Vie Nouvelle”, ebenda, S. 531-537.

${ }^{2} \mathrm{~B}$. D u v a l, L'argumentation de quelques théologiens de langue française au sujet de l'ordination des femmes, ebenda, S. 593.

${ }^{3} \mathrm{M}$. R o n de a u, Le discours du magistère papal au sujet des femmes du XIX ${ }^{e}$ siècle à nos jours, ebenda, S. 580. 
sung von Frauen zum Priesteramt befürworten würden ${ }^{4}$. Die Autorem betrachten dieses Problem von verschiedenen Standpunkten aus. Es zeichnen sich hierbei deutlich zweierlei Arten der Beweisführung ab, sowohl hinsichtlich der Möglichkeit, wie auch der Unmöglichkeit einer Zulassung von Frauen zum Priestertum. Einerseits werden soziologische, psychologische, philosophische und theologische Argumente angeführt, anderseits sucht man Anlehnung an die Texte der hl. Schrift des Neuen Testamentes.

J. Peters führt einige Gründe an, die seiner Ansicht nach, für eine Revision der traditionellen Anschauungen bezüglich des Subjektes des Sakramentes der Priesterweihe sprechen. Eingangs erwähnt er die Notwendigkeit einer Abkehr vom Konservatismus, der steif bei den traditionellen Ansichten verharrt. Zweitens führt er kulturelle Begründungen an, die heute gegen eine Diskriminierung der Frauen sprechen. Es ist nämlich das kulturelle Erbe von Frauen und Männer das gleiche. Der Katholizismus, also die Allgemeinheit der Kirche ist nach Meinung von Peters mit der biologischen Differenzierung des Menschen nicht in Einklang zu bringen und er betrachtet daher eine Zulassung der Frauen zum Priesteramt in der katholischen Kirche für erwünscht. Als dritten Grund führt er die Notwendigkeit an die unrichtige Interpretation von Texten der hl. Schrift über sogenannte Schweigen der Frau in der Kirche aufzugeben. Es können nämlich dieser Interpretation andere Texte gegenübergestellt werden (J, 4,27; Gal, 3,28), die eine derartige Diskrimination nicht zulassen. In der ersten Jahrhunderten wurden von Frauen Funktionen ausgeführt, die als priesterlich anerkannt werden könnten, wie z.B. die Erteilung der hl. Taufe, sowie die Zustellung der Eucharistie an Kranke und Häftlinge. Aufgrund der angeführten Begründungen äussert Peters die negativ formulierte These, es bestünde kein überzeugendes Argument für die ausschliessliche Zulassung von Männer zum Sakrament des Priestertums bei Ausschluss der Frauen ${ }^{5}$.

Bertille Duval, eine Vertreterin der Frauenbewegung in dieser Frage, sieht als Ursache der Reservation des sakramentalen Priestertums nur für Männer das Festhalten an dem alten Gesellschaftsmodell, das Autorität und Herrschaft dem Mann zugestand. Der Verfasserin Ansicht nach wollen die Befürworter der traditionellen Auffassung nicht zur Kenntnis nehmen, dass die Unterschiede in der Bewertung der Geschechter ihre Ursache in den damaligen sozial-kulturellen Verhältnissen und Faktoren hatten. Diese Beziehungen haben sich geändert, doch will keiner der heutigen Gegner einer Zulassung von Frauen zum Priesteramt die neue Anthropologie berücksichtigen, wonach Frauen zum Mann in persönlicher und sozialer Hinsicht ebenbürtige Partner gleicher Würde und Verantwortlichkeit darstellen. Iher meinung nach bege-

${ }^{4}$ J. Peters OCD, Gibt es für die Frau einen Platz in den kirchlichen Funktionen, „Concilium" (polonische Ausgabe) 1-10:1968 (1969) S. 216.

${ }^{5}$ Ebenda, S. 216-223. 
hen die Gegner insofern eine Inkonsequenz, als sie einerseits die Gleichberechtigung von Frau und Mann im sozialen und staatsrechtlichen Leben anerkennen, andererseits aber diese Gleichheit in der Kirche verneinen. Als Grund geben die Besorgnis über eine Maskulisation der Priesterinnen an, was zu einer Verwischung der Differenz zwischen den Geschlechtern führen könnte. Eine Revision der traditionellen Anschauungen würde, Duvals Ansicht nach, eine tiefere Erläuterung der evangelischen Sendung auf verschiedenen aufeinander folgenden Kulturebenen ermöglichen. Die Autorin ist der Meinung, dass die Theologen daraus Begründungen für die Zulassung von Frauen zur Priesterweihe entnehmen sollten ${ }^{6}$.

In der Polemik mit dem oftmals angeführten Argument, ist Duval der Ansicht, dass aus der Wahl von Männer zu den Zwölf Aposteln nicht der Ausschluss der Frau vom Priestertum gefolgert werden kann. Ihrer Meinung nach bezieht sich die Wahl des Zwölf Apostolen keineswegs auf die Institution des für Männer reservierten Priestertum, sie symbolisiert hingegen die Gesamtheit des auserwählten Volkes, als die zwölf Stämme Israels. Eben deswegen betrachteten die Apostel als notwendig nach dem Verrat des Judas ihre Anzahl wieder auf zwölf zu ergänzen. Unter der Voraussetzung dass die Zwölf nicht Männer-Aposteln gleichzustellen sind, ersteht nach Duval die begründete Frage, ob von einem bewussten Ausschluss der Frau vom sakramentalen priestertum durch Christus gesprochen werden kann. Sie fügt hierbei hinzu, dass nach einer Antwort auf diese Frage nicht im Altertum gesucht werden kann, da diese Epoche jenes Problem nicht kannte ${ }^{7}$.

Alice Gombault geht in ihren Erwägungen von der Annahme aus, dass die Domination des Mannes über die Frau die älteste Form der Domination eines Menschen über den anderen darstellt und dass darin alle anderen Formen der Diskrimination enthalten sind. Ihrer Ansicht nach wurde diese Form der Domination von der Kirche nicht nur erhalten, sondern noch sakralisiert. Das zukünftige Aufgeben dieser Diskrimination, wozu übrigens unsere Gesellschaft noch nicht vorbereitet ist, wird als Folge den schmerzlichen aber unvermeidlichen Verzicht auf den traditionellen Grundsatz der Nichtzulassung von Frauen zum Priestertum mit sich bringen. Gombault meint, dass der Anfang solcher Umgestaltungen in der Kirche schon fühlbar ist, da die Tatsachen hierzu zwingen. Die Kirche müsste das alles erwägen um dadurch die Chance einer besseren Verwirklichung evangelischer Werte in Leben zu erreichen ${ }^{8}$.

Marie J. Bérére ersieht in der Deklaration der Kongregation der Glaubenslehre vom Jahre 1970, worin die Praxis der Nichtzulassung von Frauen zum sakralen Priestertum aufrecht erhalten wird, eine Missachtung der Evolution des sozialen und geselligen Lebens im Bereich der Kirche. Die Mitwirkung der

${ }^{6}$ B. Duval, a. a. O., S. $606 \mathrm{f}$.

${ }^{7}$ Ebenda, S. $601 \mathrm{f}$.

${ }^{8}$ A. Go m ba ul t, La nouvelle condition féminin, „Le Supplément” 127: 1978 S. 494. 
Frauen am Werk der Katechisation und Liturgie wird von der Kirche zugelassen, doch wird sie in der sakramentalen Anordnung diskriminiert. Es muss deshalb, ihrer Meinung nach, wenn von der Position der Frau in der heutigen Kirche die Rede ist, weiterhin zwischen ihrer tatsächlichen und rechtlichen Lage unterschieden werden. Auf dem faktischen Gebiet erfolgen in der Kirche nach dem II Vatikanischen Konzil tiefgehende Veränderungen, es ändert sich aber nichts auf der rechtlichen Seite und die Frauen werden weiterhin zum Priesteramt nicht zugelassen ${ }^{9}$.

Die Gegner der Zulassung von Frauen zum Priestertum betrachten im Prinzip das soziologische Argument für richtig, doch wird ihm nur begrenzte Anwendbarkeit zuerkannt. Die traditionelle Anschauung über Ausschluss der Frau vom Sakrament der Priesterweihe stüzt sich nach Y. Congar auf die Tatsache der ausschliesslichen Berufung von Männer zu Aposteln, obwohl Jesu Christo tatsächlich auch Dienste von Frauen erwiesen wurden. Er gibt zu, dass die auf der Tatsache der ausschliesslichen Berufung von Männer zum Priestertum beruhende Beweisführung, sich nur auf die vergangene Welt bezieht, in der die Frau als etwas tieferstehendes und unterworfenes angesehen wurde. Infolgedessen bestand die Ansicht, dass sie keine den Männer ebenbürtige soziale Verantwortlichkeit besitze. Derartige sozial-kulturelle Umstände, schreibt Congar, treffen heute nicht zu. Darum könnte es angenommen werden, dass die Nichtzulassung von Frauen zum Priesteramt die Folge der damaligen sozialen Verhältnisse sei. Congar zieht sogar noch weitergehende Schlussfolgerung, es sei gar nicht sicher, dass der Ausschluss der Frauen vom Priesteramt dem göttlichen Gesetz entstamme. Trotz dieser Vobehalte stimmt Congar der traditionellen Ansicht zu, mit der Begründung, die Frau sei durch ihr Geschlecht tiefer bedingt als der Mann. Darum die Zulassung zum Priesterstand würde zu ihrer Vermännlichung führen ${ }^{10}$.

Nach J. Vinatier haben drei falsche Gedankengänge die bisherige Diskussion über die Möglichkeit des Priestertums der Frau gehemmt. Die erste falsche Gedankenrichtung war die ausschliessliche Erwägung des Modells der Mutter Gottes als Muster für die Frau und das Übersehen der Tatsache, dass auch andere Frauen Jesus begleiteten. Der zweite falsche Gedankengang gründete die Diskussion auf den Worten des hl. Apostel Paulus, bezüglich des Schweigens der Frau in der Kirche, unter Missachtung der Mentalität jener Epoche. Die Grundlage der dritten falschen Richtung war die Überzeugung, dass religiöse Leben selbst alle Probleme der Frauen erfüllen und lösen könnte. Vinatier gibt zu, dass vatikanische Konzil fordere einerseits Vollwertigkeit der Frau in der Welt, schweige aber andererseits über ihre Stellung in der Kirche. Der Autor lässt sogar die Möglichkeit zu, dass im Evangelium nicht

${ }^{9}$ M.J. B é ré r e, Symbolisme chrétien et situation ecclésiale des femmes, ebenda, S. 613.

${ }^{10}$ B. Duval, a. a. O., S. $594-596$. 
alle Arten der Dienste durch Christus den Herrn ein für alle Male bestimmt worden seien. Er ist deshalb der Meinung, dass in Verbindung mit der apostolischen Tradition den Frauen in der Kirche der Platz zu Dienstleistungen gemäss den Erfordernissen der heutigen Zeit zu öffnen sei.

Nach seiner Ansicht haben nämlich die Frauen dieselbe Art der Mission erhalten, wie die Zwölf und der hl. Apostel Paulus. Eine Gruppe von Frauen begleitetet Jesus gleichzeitig mit den Aposteln. Eben der Samariterin und nicht den Aposteln erteilt Jesus die Mission der Evangelisierung der Samariter. Ähnlich tat Er es nach der Auferstehung, als Er zuerst den Frauen erschien und ihnen die Mission erteilte die Apostel zu benachrichtigen. In seiner Konklusion verengt jedoch Vinatier diese Dienstleistungsmission der Frauen in der Kirche höchstens zum Diakonat, als den Fähigkeiten der Frau entsprechender Würde. Das Modelle des Priesteramtes betrachtet er als männlich in dem Grad, dass die Frau durch dessen Übernahme ihre Weiblichkeit einbüssen würde. Vinatier erläutert näher nicht, worauf der Verlust der Weiblichkeit durch Übernahme der Priesterweihe durch Frau beruhen sollte ${ }^{11}$.

J. Galot versucht auf andere Weise die traditionelle Stellung der Nichtzulassung der Frau zur Priesterweihe zu begründen. Nach seiner Ansicht sollten, sobald Gottes Sohn als Mensch männlichen Geschlechtes zur Welt kam, auch seine Priester Männer sein. Das Priesteramt impliziert für die Kirche eine Hierarchie der Autorität und die Männer besitzen eine höchere Befähigung zum Regieren als die Frauen. Nach Golt wäre deshalb das Priesteramt ausschliesslich für Männer zu resevieren. Diese Beweisführung ist aber auch für Galot selbst nicht überzeugend und er stützt den Ausschluss der Frauen vom Priesteramt auf die a priori und a posteriori ohne Beweis angenommen Hypothese, dass Priesteramt wäre aufgrund eines bewussten Willensaktes Christi für Männer reserviert. Dieser Entschluss Christi hat seine Wurzeln im grossen Geheimnis der Inkarnation und in dem Erlösungsplan Gottes. Um seine Behauptung zu begründen beruft sich Galot auf das Zeugnis der hl. Schrift, nämlich auf die Texte von der Auswahl der Zwölf (Lk, 6, 12-16), ihre Zulassung zur Eucharistie (Mt, 26, 20-29), von der Erteilung der Macht zum Ablass der Sünden an die Aposteln ( $\mathrm{J}, 20,22-23)$ und ihrer Entsendung in die gesamte Erde mit der Mission der Evangelisierung der Welt (Mt, 28, 18-20; Mk, 16, 15-18) ${ }^{12}$.

L. Bouger versucht auf ziemlich originelle Weise den Ausschluss der Frauen vom Priesteramt zu begründen. Als Ausgangspunkt wählt er das Geheimnis der Hl. Dreifaltigkeit an. Gott Vater unterscheidet sich vom Sohn durch die Vaterschaft. Auf der Erde wird die Tat der Vaterschaft durch Männer verwirklicht und die Vaterschaft ist die wesentliche Bekundung das Mannes. Es stellt somit der Mann, wenn auch auf unvollkommene Weise, die Vaterschaft

${ }^{12}$ Ebenda, S. $599 \mathrm{f}$. 
Gott-Vaters dar. Die Frau hingegen bildet auf Grund ihrer Jungfräulichkeit nur den Reflex Gott-Vaters. Die Mutterschaft bezeugt die Fruchtbarkeit der gesamten Schöpfung durch den Hl. Geist. Die hl. Schrift stellt das Verhältnis Gottes zur Schöpfung und Christus zur Kirche durch das Symbol der auserwähIten Braut dar. Nach Bougers Ansicht führt dies zur Formulierung von Gegensätzen, die eine Reservation des Priesteramtes für Männer begründen. Es sind die Gegensätze: Transcendenz-Immanenz, Autorität-Fürsprache, Vaterschaft-Mutterschaft, Mann-Weib. Der Mann representiert die Transcendenz, Autorität und Vaterschaft, die Frau hingegen die Immanenz, Fürsprache und Mutterschaft. Es soll somit die Frau das Vorbild der Frau-Gottesmutter nachahmen ${ }^{13}$.

Es ergibt sich jetzt die grundsätzliche Frage, ob derartige, oder ähnliche Argumente sowohl für, wie auch gegen die Möglichkeit der Zulassung von Frauen zur Priesterweihe Beweiswert besitzen. Manche Autoren und insbesondere Autorinnen unterstreichen die Veränderung der sozial-kulturellen Verhältnisse in unserer Zeit, was ihrer Ansicht nach sich auf die Lösung des Problems des Priestertums von Frauen in der gegenwärtigen katholischen Kirche auswirken müsste. Sie äussern die Meinung, es könnte und müsste heute das geändert werden, was zu Christi Zeiten nicht möglich war. M. Rondeau meint, dass diese traditionelle Anschauung gegenüber der Frau noch gegenwärtig in der Kirche erhalten ist und ihr den Zutritt zum Sakrament der Priesterweihe blokiert ${ }^{14}$.

Es ist tatsächlich wahr, dass die Frauen lange Zeit zum sozialen und staatlichen Leben nicht zugelassen waren, was wegen der sozialen Gründen als natürlich betrachtet wurde. Es ist jedoch dieses Argument nicht ausreichend, um auf dessen Grund die traditionelle Haltung der Kirche hinisichtlich des Priesterstandes der Frauen zu modifizieren. Wir wissen nämlich nicht, und niemand kann das wissen, aus welchen Gründen Christus nur Männer zu Aposteln und als dessen Folge zu Priestern beruf. Jedoch ist es ganz sicher, dass sich Christus nicht nach sozial-kulturellen Voraussetzungen richtete. Der Herr Jesus Christus richtet sich nicht nach dieser oder jener menschlichen Tradition, sondern nach dem Willen des Vaters im Himmel und brach Ihm folgend diejenigen bestehenden Anschauungen, die mit seiner Lehre nicht im Einklang waren. Es wäre zumindest erniedrigend Christo zuzuschreiben, dass Er in eiber für die Kirche so wesentlichen Angelegenheit wie das Sakrament der Priesterweihe, sich den zu seinen Zeiten herrschenden jüdischen Bräuchen anpasste. Ganz im Gegenteil war eben Christus, der durch Aufwertung der Frau sie im Bereich der Erlösung und des Königreiches Gottes dem Mann gleichstellte und

${ }^{13}$ Ebenda, S. $603-605$.

${ }^{14}$ M. Rondeau, a. a. O., S. 581. 
ihr somit den Weg zur völligen Gleichheit mit dem Mann auf sozialer und staatlicher Ebene öffnete.

Die Argumente von Gegner einer Zulassung der Frauen zum Priesterstand sind ähnlich zu beurteilen. Es findet sich in der hl. Schrift keinerlei direkte Aussage, die das Priesteramt den Männer reservieren und den Frauen vorenthalten würde. Analogien zur Hl. Dreifaltigkeit und dem männlichen Geschlecht Christi bilden eine unzulässige Übertragung menschlicher Auffassungsweise auf den unbegreiflichen und als Gott geschlechtslosen Gott. Ein übertriebenes Unterstreichen der Rolle des Geschlechtes und die Berufung auf das Modell der Mutter Gottes für die Frau bildet kein Argument, da es ein anderes Problem als das Priestertum der Frauen betrifft. Die hl. Schrift unterstreicht, es gäbe keine Unterscheidung zwischen Mann und Frau in der Kirche Christi (Gal, 3, 28), spricht von einem allgemeinen Priestertum der Gläubigen (1 P,2, 9ff), doch gibt dies keine Grundlage zur Lösung des Problems des sakramentalen Priestertums hinsichtlich des Geschlechtes.

Alle angeführten pro und contra Argumente ergeben keine Lösung des Problems der Zulassung oder Ausschlusses der Frauen vom Sakrament der Priesterweihe in der katholischen Kirche. Es sind dies nur Hinweise, Wünsche, Suggestionen, doch keine Beweise und es ist deshalb nach einem anderen Grund zur Lösung dieser Frage zu suchen. Es ist vorerst klarzustellen, dass dem Wort „Priestertum” eine zweifache Bedeutung zugeschrieben werden kann, je nach dem wir es in der natürlichen oder übernatürlichen Ebene festsetzen. Bei Versuchen einer Argumentation kommt oftmals zu einer Verwischung dieser zwei Ebenen, was eher Verwirrung als eine Lösung des Problems als Folge erbringt. Auf natürlicher Ebene ist die Lösung klar und einfach. Frau und Mann sind auf allen Gebieten des Lebens gleichgestellt, sowohl in sozial-kultureller wie in staatsrechtlicher und religiöser Hinsicht. In Anlehnung an die natürliche Denkweise sind die Rechte der Frau und des Mannes zum Priestertum gleich. Es gibt auf natürlicher Ebene keine Begründung für den Ausschluss der Frau vom natürlichen Priestertum. Dafür spricht ebenfalls die Tatsache, dass seit frühester Zeit in den natürlichen Religionen Priesterinnen auftreten. Es beginnen zwar auch in den abgetrennten christlichen Kirchen in Einzelfällen Priesterinnen zu erscheinen, doch sind diese natürliche und nicht sakramentale Priesterinnen.

Das Problem des sakramentalen Priestertums in der katholischen Kirche kann ausschliesslich auf der übernatürlichen, also nur vom Willen Gottes abhängigen Ebene erörtet werden. Es besteht nämlich dieses Problem auf dem Gebiet der geoffenbarten und nicht natürlichen Religion. Die geoffenbarte Religion ist die Sprache Gottes an die Menschen und es entspringen hieraus gewichtige Konsequenzen bezüglich unseres Problems. In Beantwortung der geoffenbarten Sprache kann der Mensch nur sie Stellung eines Hörenden annehmen und nicht eines Diskutanten und für seine Rechte, die er übrigens nicht 
besitzt, eintretenden Partners. Es ist daher deutlich festzustellen, dass die Frage des sakramentalen Priestertums der Frauen in keinerlei Verbindung zu diesen oder jenen Problem sozialer, kultureller, psychologischer, biologischer, philosophischer oder anderer Art steht. Die Zulassung oder der Ausschluss der Frauen aus der Priesterweihe steht in keiner Beziehung zur Stellung der Frau in der Welt sowohl des Altertums zur Zeit Christi, wie den heutigen Zeiten. Die Frage einer Zulassung von Frauen zum sakramentalen Priesterstand ist nicht Gegenstand einer Vereinbarung, einer Abstimmung der Gläubigen, des Priesterkollegiums und sogar auch nicht des Magisterium Ecclesiae. Es ist dies auch keine Angelegenheit der reinen kirchlichen Legislative, der Rechtsvorschriften, die man abändern kann, oder in die die Korrekturen oder Veränderungen eingeführt werden können.

Es kann die scheinbar paradoxale Bahauptung aufgestellt werden, dass wir und niemand wissen können, was Christus dachte und zu erreichen beabsichtigte, als Er sich entschloss zu Aposteln und Priester Männer zu berufen. Niemand weiss und kann nicht wissen, weshalb Er die Ihm begleitenden Frauen überging. Darum sind wir gezwungen eine andere Grundlage für die Lösung dieses Problems zu suchen. Den Ausgangspunkt unserer Erwägungen kann nur das in der Tat Christi geäusserte Faktum bilden, dass zum sakramentalen Priestertum zwölf Männer-Apostel berufen wurden und seine jungfräuliche Mutter und alle Ihn begleitende Frauen unberücksichtigt blieben. Unerfassbar sind für uns die Gründe, weshalb Christus so und nicht anders entschloss, doch zu erfassen ist für uns die Tatsache dieses und nicht anderes Tuns Christi.

Das Problem der Nichtzulassung von Frauen zum sakramentalen Priestertum in der katholischen Kirche ist also erstens nur auf der übernatürlichen Ebene zu lösen, zweitens nur durch Erhören der Offenbarung Gottes und drittens indem man die Tat Christi des Herrn als Äusserung seines Willens und als göttliches Mysterium entgegennimmt. Das sakramentale Priestertum in der katholischen Kirche ist seiner Entstehung und seinem Wesen nach übernatürlich. Als solches ist die Priesterwürde eine dem Menschen umsonst zu Teil geworden Gnade Gottes. Sie ist somit kein Objekt des Verdienstes, etwas was der Mensch verlangen und verdienen könnte. Die Texte der hl. Schrift besagen deutlich: „Nicht ihr habt mich erwählt, sondern ich habe euch erwählt und euch dazu bestellt, dass ihr hingeht und Frucht bringt" $(\mathrm{J}, 15,16)$.

Diese Worte Christi des Herrn, als Ausdruck Seiner Tat, bilden die einzige Grundlage zur Lösung des Problems der Zulassung oder Nichtzulassung von Frauen zum Sakrament der Priesterweihe. Zur sakramentalen Priesterwürde, die eine übernatürliche Gnade und Gabe darstellt, kann deshalb niemand, weder Mann, noch Frau und auch kein Engel selbst ein Anrecht beanspruchen. Niemand darf sich als übergangen fühlen, wenn ihm diese Gnade nicht zu Teil wird. In dieser Hinsicht ist der Mensch kein Partner und kein gleichberechtig- 
ter Gegensprecher im Dialog mit Gott. Verstandesmässige Argumente bilden hier gar keinen Beweis (Rom, 9, $15 \mathrm{ff}$ ).

Daraus folgt, dass einzig entscheidend bei der Lösung des Problems des sakramentalen Priesteramtes für Frauen das Tun Christi des Herrn ist, und diese Tat ist klar und eindeutig: Er bestellte die Apostel zu Priestern und verpflichtet sie dieses Sakrament ihren Nachfolgern zu überwiesen. Die Zulassung nur von Männer zum Priesteramt ist somit nicht eine Diskriminierung der Frauen, sondern eine derartige Tat Christi. Der Herr Jesus machte nicht einmal seine Mutter, die frei war von der Erbsünde, zur Priesterin. Das sakramentale Priestertum steht vor uns als Mysterium der freien Tat $\mathrm{Ch}$ risti. Bei Annahme dieser Voraussetzung wird das Problem der Teinahme von Frauen am sakramentalen Priestertum der katholischen Kirche nur auf der übernatürlichen Ebene gelöst, in Anlehnung an d a s k on k re te Vorge hen Christi.

Als nächste tritt die Frage auf, ob eine Möglichkeit besteht die Folgen dieser Tat Christi zu ändern, ob also die Kirche diesen Brauch durch Zulassung von Frauen zur Priesterweihe abzuändern vermag. Es scheint, dass nur eine Antwort möglich ist. Wenn das Priestertum eine übernatürliche Gabe Gottes bedeutet, über die Jesus sich ausdrücklich äusserte und die ganze Intiative ausschlesslich von Ihm stammt, da Er selbst den Menschen ohne dessen Verdienst beruft, dann kann eine Änderung dieses Praxis nur durch e in e d e u t liche Anweisung Christi des Herrn erfolgen. Ohne den ausdrücklichen Willen Christi vermag das Magisterium Ecclesiae an den der Kirche überwiesenen Glaubensdeposit nichts zu ändern.

Die öffentliche Offenbarung fand ihr Ende mit dem Tode des letzten Apostels, es kann deshalb keinerlei Änderung eintreten in der Tat Christi nur Männer zum Priesterstand zu berufen. Private Offenbarungen und rationale begründungen besitzen in diesem Fall gar keine Exekutivkraft. So wie die Worte Christi man nicht ändern kann, so auch die Taten. Es wäre somit die Zulassung von Frauen zum sakramentalen Priestertum eine eigenwillige Änderung des Tuns Christi in einem für die Kirche wesentlichen Bereich, und das überschreitet die Kompetenz der Kirche. Hier liegt eben die grundlegende Ursache für die Unabänderlichkeit der kirchlichn Praxis der Nichtzulassung von Frauen zur Priesterweihe. Es ist unzulässig sich auf die Beispiele der abgetrennten Kirchen zu berufen, da jene Frauen zum Priesteramt nicht Kraft der Autorität Christi des Herrn, sondern auf Grund arbitraler menschlichen Entscheidungen berufen sind. Ein derartiges Priesteramt ist kein sakramentales Priestertum, da ja nicht die Gemeinde der Gläubigen und nicht die Pastoren, sondern Christus selbst der Ursprung des Sakramentes der Priesterweihe ist.

Zusammenfassed können wir sagen, dass wir nicht wissen, was für Absichten Christus der Herr hatte und weswegen Er nur Männer zu Priestern be- 
stellte. Wir wissen nicht, ob die Priesterwürde den Männer iure Divino vorbehalten ist, wissen ferner nicht, ob nur Mann oder auch die Frau zum Empfang des Sakramentes der Priesterweihe fähig ist. Hingegen wissen wir, was Christus getan hat und es ist deshalb die Tat Christi in dieser Hinsicht en ts cheidend. Wir sind nicht nur zur Exegese der Worte, sondern auch zur Exegese der Taten Christi verpflichten. Die Kirche kann nicht anders vorgehen als Christus vorging und kann nicht ändern was Christus getan hat. Das Tun Christi ist regula procedendi der Kirche.

Reicht man tiefer zur mystischen Bedeutung der Tat Christi, ersehen wir hier ein Mysterium Dei, das sich darin äussert, dass die Frau-Mutter der Welt den Gottesmenschen Jesus Christus gebracht hat (was ein Mann nicht vollbringen kann), den die Männer-Priester in sakramentaler Gestalt (was eine Frau nicht vollbringen kann) an die Gesamtheit der Erdbewohner übertragen. Das, was die Frau-Mutter im Bereich der Erlösungstat begann, wird von den Männer-Priestern weiter geführt. 